\title{
A NOTE ON KRYLOV-TSO'S PARABOLIC INEQUALITY
}

\author{
LUIS ESCAURIAZA
}

(Communicated by Barbara L. Keyfitz)

\begin{abstract}
We show that if $u$ is a solution to $\sum_{i, j=1}^{n} a_{i j}(x, t) D_{i j} u(x, t)-$ $D_{t} u(x, t)=\phi(x)$ on a cylinder $\Omega_{T}=\Omega \times(0, T)$, where $\Omega$ is a bounded open set in $\mathbf{R}^{n}, T>0$, and $u$ vanishes continuously on the parabolic boundary of $\Omega_{T}$. Then the maximum of $u$ on the cylinder is bounded by a constant $C$ depending on the ellipticity of the coefficient matrix $\left(a_{i j}(x, t)\right)$, the diameter of $\Omega$, and the dimension $n$ times the $L^{n}$ norm of $\phi$ in $\Omega$.
\end{abstract}

\section{INTRODUCTION}

Let $L=\sum_{i, j=1}^{n} a_{i j}(x, t) D_{i j}-D_{t}$ be a parabolic operator with bounded measurable coefficients defined on a cylinder $\Omega_{T}=\Omega \times(0, T)$, where $\Omega$ is a bounded open set in $\mathbf{R}^{n}, T$ is a positive real number, $a_{i j}(x, t)=a_{j i}(x, t)$ for all $x$ in $\Omega$, real $t$, and $i, j=1, \ldots, n$, and where there exists $\lambda>0$ so that the following holds,

(1) $\lambda^{-1}|\xi|^{2} \geq \sum_{i, j=1}^{n} a_{i j}(x, t) \xi_{i} \xi_{j} \geq \lambda|\xi|^{2} \quad$ for all $x \in \Omega, t \in \mathbf{R}$, and $\xi \in \mathbf{R}^{n}$.

In $[5,6]$ it is shown that if $u \in C\left(\bar{\Omega}_{T}\right)$ is smooth in $\Omega_{T}$ with $u=0$ on the parabolic boundary of $\Omega_{T}$, i.e., $\partial_{p} \Omega_{T}=\partial \Omega \times[0, T) \cup \Omega \times\{0\}$ and $|L u| \leq$ $f(x, t)$ on $\Omega_{T}$ the following holds,

$$
\sup _{\Omega_{T}}|u| \leq C \delta(\Omega)^{n /(n+1)}\|f\|_{L^{n+1}\left(\Omega_{T}\right)},
$$

where $\delta(\Omega)$ denotes the diameter of $\Omega$ and $C$ is a constant depending on $\lambda$ and the dimension $n$. In this paper we will observe that a more careful analysis of the argument given by Kai-Sing Tso in [5] shows that when the above conditions are satisfied and the function $f(x, t)$ does not depend on time, that is, when $f(x, t)=\phi(x)$ then

$$
\sup _{\Omega_{T}}|u| \leq C \delta(\Omega)\|\phi\|_{L^{n}(\Omega)},
$$

Received by the editors September 4, 1990 and, in revised form, January 28, 1991.

1980 Mathematics Subject Classification (1985 Revision). Primary 35A.

The author is partially supported by the National Science Foundation Grant \#NSF/DMS 8421377-03. 
and where the constant $C$ has the same dependence as before. We observe that the classical Pucci-Aleksandrov inequality for solutions to elliptic operators can be recovered from the above inequality (see $[1,2,7])$. For if $S=$ $\sum_{i, j=1}^{n} a_{i j}(x) D_{i j}$ is an elliptic operator satisfying $a_{i j}(x)=a_{j i}(x)$ for all $x$ in $\Omega$ and $i, j=1, \ldots, n$, and where there exists $\lambda>0$ so that

$$
\lambda^{-1}|\xi|^{2} \geq \sum_{i, j=1}^{n} a_{i j}(x) \xi_{i} \xi_{j} \geq \lambda|\xi|^{2} \quad \text { for all } x \in \Omega \text { and } \xi \in \mathbf{R}^{n},
$$

and $v \in C(\bar{\Omega})$ is smooth in $\Omega$ with $v=0$ on the boundary of $\Omega$ and $|S v(x)| \leq h(x)$ in $\Omega$, the function $u(x, t)=v(x) t$ satisfies $|L u(x)| \leq T h(x)+$ $|v(x)|$, where $L$ is the parabolic operator given by $L=S-D_{t}$. Hence, from (3) we get

$$
\sup _{\Omega}|v| \leq C \delta(\Omega)\|h\|_{L^{n}(\Omega)}+T^{-1} \delta(\Omega)|\Omega|^{1 / n} \sup _{\Omega}|v|,
$$

where $|\Omega|$ denotes the $n$-dimensional Lebesgue measure of $\Omega$. After letting $T$ tend to infinity we obtain the maximum of $v$ over $\Omega$ controlled by the $L^{n}$ norm of $h$ in $\Omega$.

\section{PROOF OF THE INEQUALITY}

Theorem. Let $u \in C\left(\bar{\Omega}_{T}\right)$ and smooth in $\Omega_{T}$ with $u=0$ on $\partial_{p} \Omega_{T}$ and $|L u| \leq$ $\phi(x)$ on $\Omega_{T}$ for some $\phi$ lying in $L^{n}(\Omega)$, and where $L$ is a parabolic operator satisfying (1). Then, there is a constant $C$ depending only on $\lambda$ and $n$ so that (3) holds.

Proof. We may assume that for some $x_{0}$ in $\Omega$ and $0<\tau \leq T, u\left(x_{0}, \tau\right)=$ $\sup _{\Omega_{T}}|u|$. From the argument in the proof of Proposition 2.1 in [5]

$$
u\left(x_{0}, \tau\right)^{n+1} \leq C \delta(\Omega)^{n} \iint_{A_{u}}\left|\operatorname{det}\left(D_{i j} u(x, t)\right) D_{t} u(x, t)\right| d x d t
$$

where $A_{u}=\left\{(x, t) \in \Omega \times[0, \tau)\right.$ : there exists $\xi$ in $\mathbf{R}^{n}$ so that $u(y, s) \leq$ $u(x, t)+\xi(y-x)$ for all $y \in \Omega$ and $0 \leq s \leq t\}$ and $C$ depends only on dimension $n$. It is shown in [5] that at points $(x, t)$ lying in $A_{u}$ the matrix $\left(D_{i j} u(x, t)\right)$ is nonpositive and $D_{t} u(x, t)$ is nonnegative. Since $|L u| \leq \phi$ on $\Omega_{T}$ we have $-\sum_{i, j=1}^{n} a_{i j}(x, t) D_{i j} u(x, t) \leq \phi(x)$ on $A_{u}$. On the other hand, the symmetry of the coefficient matrix $\left(a_{i j}(x, t)\right)$ and the nonpositiveness of the Hessian of $u$ imply

$$
\begin{aligned}
\left|\operatorname{det}\left(D_{i j} u(x, t)\right)\right| & =-\operatorname{det}\left(D_{i j} u(x, t)\right) \\
& \leq \lambda^{-n}\left(-\sum_{i, j=1}^{n} a_{i j}(x, t) D_{i j} u(x, t)\right)^{n} \leq \phi(x)^{n}
\end{aligned}
$$

for all $(x, t)$ in $A_{u}$. Hence,

$$
u\left(x_{0}, \tau\right)^{n+1} \leq C \lambda^{-n} \delta(\Omega)^{n} \iint_{A_{u}} \phi(x)^{n} D_{t} u(x, t) d x d t .
$$

If $H$ denotes the projection of $A_{u}$ into $\Omega$ and $I(x)=\left\{t \in[0, \tau]:(x, t) \in A_{u}\right\}$ we have

$$
u\left(x_{0}, \tau\right)^{n+1} \leq C \lambda^{-n} \delta(\Omega)^{n} \int_{H} \phi(x)^{n} \int_{I(x)} D_{t} u(x, t) d t d x
$$


Observe that if $(x, t) \in A_{u}$ and $\xi$ is as in the definition of the set $A_{u}$ we have $|\xi| \leq u(x, t) / d(x, \partial \Omega)$. To see this let $y=x-\sigma \xi /|\xi|$, where $\sigma \geq d(x, \partial \Omega)$ is a positive number so that $y$ lies in the boundary of $\Omega$. Since $u(y, t)=$ 0 it follows from the definition of $A_{u}$ that $0 \leq u(x, t)-\sigma|\xi|$. From this fact it follows that the set $I(x)$ is closed relative to $[0, \tau]$ for each $x$ in $H$. Therefore, $[0, \tau] \backslash I(x)$ can be written as a disjoint union of intervals $\left\{I_{j} \mid j \geq 1\right\}$ and where each $I_{j}$ is an interval having one of the following forms: $[0, a)$, $(b, c)$, or $(d, \tau]$ with $a \leq b<c \leq \tau$. From the definition of $I(x)$ and when $I_{j}$ has the form of one of the two first intervals, the integral $\int_{I_{j}} D_{t} u(x, t) d t$ is nonnegative. Among the intervals $I_{j}$ there is at most one of them of the form $(d, \tau]$. Hence,

$$
u(x, \tau)=\int_{0}^{\tau} D_{t} u(x, t) d t \geq \int_{I(x)} D_{t} u(x, t) d t+u(x, \tau)-u(x, d) .
$$

The above argument shows that for all $x$ in $H$ we have

$$
\int_{I(x)} D_{t} u(x, t) d t \leq u\left(x_{0}, \tau\right)
$$

which together with (4) implies the theorem.

Finally, I would like to remark some new results that are consequences of the above theorem. In [8, Theorem 3.2] Ural'zeva and Ladyzenskaya show that when $\Omega$ is a $C^{2}$ domain and $u$ denotes the solution to

$$
\begin{cases}L u=f(x, t)+\phi(x) & \text { on } \Omega_{T} \\ u=0 & \text { on } \partial_{p} \Omega_{T}\end{cases}
$$

then there are constants $C>0$ and $\alpha \in(0,1)$ with $C$ and $\alpha$ depending on $\lambda, n, T, p$, and the $C^{2}$ character of the boundary of $\Omega$ so that

$$
\left\|\frac{\partial u}{\partial n}\right\|_{L^{\infty}\left(S_{T}\right)}+\left\|\frac{\partial u}{\partial n}\right\|_{C^{\alpha, \alpha / 2}\left(S_{T}\right)} \leq C\left\{\|f\|_{L^{p}\left(\Omega_{T}\right)}+\|\phi\|_{L^{n+1}(\Omega)}\right\}
$$

for all $p>n+2$, where $S_{T}=\partial \Omega \times(0, T)$ and $\partial u / \partial n$ denotes the normal derivative of $u(\cdot, t)$ on the boundary of $\Omega$. By the parabolic analog of the methods used in [3] and (3) the above estimate can be improved in the following way: there exists $C$ and $\alpha$ depending on $\lambda, n, T, p, q$, and the $C^{2}$ character of the boundary of $\Omega$ so that

$$
\left\|\frac{\partial u}{\partial n}\right\|_{L^{\infty}\left(S_{T}\right)}+\left\|\frac{\partial u}{\partial n}\right\|_{C^{a, \alpha / 2}\left(S_{T}\right)} \leq C\left\{\|f\|_{L^{p}\left(\Omega_{T}\right)}+\|\phi\|_{L^{q}(\Omega)}\right\}
$$

for all $p>n+2$ and $q>n$. 
Another interesting consequence of (3) is that if $g(x, t, y, s)$ denotes the Green's function for the operator $L$ in the cylinder $\Omega \times \mathbf{R}$, the function $w(x, t, \cdot)$ given by

$$
w(x, t, y)=\int_{-\infty}^{t} g(x, t, y, s) d s
$$

lies in the class of weights $B_{n / n-1}(\Omega)$ independently of $(x, t) \in \Omega \times \mathbf{R}$. That is, there exists a constant $C$ depending only on $\lambda$ and $n$ so that for all cubes $Q_{r}$ of diameter $r$, with sides parallel to the coordinate axes and whose doubled concentric cube $Q_{2 r}$ is contained in $\Omega$ the following holds:

$$
\left\{r^{-n} \int_{Q_{r}} w(x, t, y)^{n /(n-1)} d y\right\}^{(n-1) / n} \leq C r^{-n} \int_{Q_{r}} w(x, t, y) d y .
$$

This last claim can be proved by the parabolic analog of the methods used in [4, Lemma 2.0, Theorems $2.1,2.2]$ to show that the Green's function $G(x, \cdot)$ on $\Omega$ of an elliptic operator $S$ as before lies in $B_{n / n-1}(\Omega)$.

\section{REFERENCES}

1. A. D. Aleksandrov, Majorization of solutions of second order linear equations, Vestnik Lenningrad Univ. Math. 21 (1966), 5-25; English transl., Amer. Math. Soc. Transl. 68 (1968), 120-143.

2. I. Ya. Bakel'man, Theory of quasilinear elliptic equations, Siberian Math. J. 2 (1961), 179186.

3. B. Barcelo, L. Escauriaza, and E. Fabes, Gradient estimates at the boundary for solutions to nondivergence elliptic equations, Contemp. Math., vol. 107, Amer. Math. Soc., Providence, R.I., 1990, pp. 1-12.

4. E. Fabes and D. Stroock, The $L^{p}$ integrability of Green's functions and fundamental solutions of second order elliptic and parabolic equations, Duke Math. J. 51 (1984), 977-1016.

5. Kai-Sing Tso, On an Aleksandrov-Bakel'man type maximum principle for second order parabolic equations, Comm. Partial Differential Equations 10 (1985), 543-553.

6. N. V. Krylov, Sequences of convex functions and estimates of the maximum of the solution of a parabolic equation, Siberian Math. J. 17 (1976), 226-236.

7. C. Pucci, Limitazioni per soluzioni di equaziones ellittiche, Ann. Mat. Pura Appl. 161 (1966), $15-30$.

8. N. N. Ural'zeva and O. A. Ladyzhenskaya, $A$ survey of results on the solvability of boundary value problems for second order uniformly elliptic and parabolic quasilinear equations having unbounded singularities, Russian Math. Surveys 41 (1986).

Department of Mathematics, University of Chicago, 5734 University Avenue, Chicago, Illinois 60615 